\title{
Safety Evaluation of Polyethylene Terephthalate Chemical Recycling Processes
}

\author{
Frank Welle
}

check for

updates

Citation: Welle, F. Safety Evaluation of Polyethylene Terephthalate Chemical Recycling Processes. Sustainability 2021, 13, 12854. https://doi.org/10.3390/su132212854

Academic Editor: Rajeev Bhat

Received: 24 October 2021

Accepted: 17 November 2021

Published: 20 November 2021

Publisher's Note: MDPI stays neutral with regard to jurisdictional claims in published maps and institutional affiliations.

Copyright: (C) 2021 by the author. Licensee MDPI, Basel, Switzerland. This article is an open access article distributed under the terms and conditions of the Creative Commons Attribution (CC BY) license (https:// creativecommons.org/licenses/by/ $4.0 /)$.
Fraunhofer Institute for Process Engineering and Packaging (IVV), Giggenhauser Straße 35, 85354 Freising, Germany; welle@ivv.fraunhofer.de

\begin{abstract}
Polyethylene terephthalate (PET) is one of the main packaging materials for beverage bottles. Even if this polymer is good to recycle, mechanical recycling processes need a well-sorted input fraction. For less-sorted PET packaging, or even non-food input sources, chemical recycling seems to be a solution to increase PET recycling. For post-consumer recyclates in packaging applications, it is essential that the safety of the recyclates is guaranteed, and the consumers' health protected. For mechanical recycling processes, evaluation criteria are already established. For chemical recycling processes, however, such evaluation criteria are only roughly available. This study evaluated the safety of the chemical recycling process similar to the approach of the European Food Safety Authority (EFSA). However, due to the lack of information about the contamination level of the input materials for the chemical recycling process, the evaluation was adapted. In addition, the evaluation should be performed separately for the depolymerisation and for the repolymerisation steps. However, due to the high cleaning efficiencies of both steps, the evaluation can focus on the repolymerisation. This simplifies the assessment of the chemical recycling processes considerably.
\end{abstract}

Keywords: circular economy; polyethylene terephthalate beverage bottles; chemical recycling; food law compliance; diffusion modelling

\section{Introduction}

Polyethylene terephthalate (PET) is one of the main packaging materials, especially for beverages bottles. In total, 71\% of the PET material in Europe was used for PET bottles, 19\% was used for tray and blisters, $6 \%$ for flexible packaging, and $4 \%$ for others, which was an amount 5.3 Mio. $\mathrm{t}$ in 2018 [1]. The global PET market for packaging was around $22 \mathrm{Mio} . \mathrm{t}$ per year in 2019, with an annual growth rate of about 4\% [2]. From a global perspective, a major fraction of used PET bottles are still going into landfill or incineration. As a nonbiodegradable polymer, PET plays a crucial role as one of the most significant sources of accumulated waste in the landfill [3]. On the other hand, PET is the main packaging material for mechanical recycling, and several recycling processes have been established for several years [4]. However, mechanical recycling of PET beverage bottles requires a well-sorted and clean input fraction in order to prevent the recyclate containing bottles from hazardous contamination [5-7]. Such well-sorted input fractions are not available in every country. On the other hand, PET is used not only for the production of beverage bottles. PET is also used to make fibers, sheets, and films, which are used in electronics, automotive parts, carpets, photographic applications, and textiles, etc. [3,8-10]. Due to the heterogeneity of these applications and the lack of suitable recollection systems, these PET applications are excluded from mechanical recycling at the moment [11].

Chemical recycling seems to be an alternative to mechanical recycling, which makes less pure or less controlled recollected packaging waste available for circular economy [7]. Chemical recycling of PET has evolved several chemical recycling methods, namely, hydrolysis, methanolysis, glycolysis, aminolysis, and ammonolysis. These depolymerisation reactions require catalysts and typically heat treatment $[1,6,9]$. Chemical recycling involves 
depolymerisation of the polymer backbone down to smaller segments such as monomers, oligomers, and gaseous intermediates. After purification, these reaction products can be used for repolymerisation into new packaging materials. Typically, chemical recycling of PET leads to monomers such as terephthalic acid (TPA), dimethyl terephthalate (DMT), bis(2-hydroxyethyl) terephthalate (BHET), and ethylene glycol (EG), or to the oligomers of PET depending on the applied chemical recycling process and extent of the degradation process.

In the end, both chemical and mechanical recycling processes are needed to achieve circular economy of PET bottles [7]. The circular economy might only work if chemical and mechanical recycling are used side by side. Mechanical recycling can provide high quality recyclates. However, the input material for the recycling processes needs to be well-sorted. Recycling from bottle-to-bottle requires this high quality and low contaminated input materials, otherwise consumer safety cannot be guaranteed $[4,5]$. These very high-quality recyclates are then also of interest for other industries or applications. For example, in Germany, PET bottles are recollected via a deposit system. A total of $98 \%$ of bottles that are brought on the market are recollected and recycled, but only 30\% go back into new PET bottles [12]. This means that 70\% of the recollected and recycled PET bottles are used in other products such as films, sheets, trays, fibres, strappings, or floor coverings. Due to the fact that efficient return systems are currently only established for PET bottles, this also means that $70 \%$ of recycled PET bottles disappear from the balance sheet and are no longer recycled. Only 30\% if the recycled PET bottle flakes are available for closed-loop recycling [12]. From a European perspective, the situation is even worse. The recollection rate in Europe is about $60 \%$, and only $11 \%$ of the post-consumer PET recyclates are going back into beverage bottles [1]. Thus, there is a lot of competition here between the applications regarding the input materials (bottle flakes) for recycling.

Chemical recycling can close this gap by making lower quality input materials available for recycling, which are currently not available for closed-loop recycling. For example, PET sheets, fibres, and flooring can be used as input materials for chemical recycling to produce monomers that can be re-used for PET bottle manufacturing. Chemical recycling is an option for recycling of highly contaminated input materials that cannot be recycled by mechanical recycling processes. Therefore, chemical recycling should not considered as a competitive recycling option to mechanical recycling of PET.

For high quality application of post-consumer recyclates, it is essential that the safety of the recyclates is guaranteed. For mechanical recycling processes, evaluation criteria are already established. The European Food Safety Authority (EFSA) published their evaluation principles for mechanical recycled PET, which is based on the following key issues [13]:

- Contamination level of the input of the recycling process;

- Cleaning efficiency of the applied mechanical recycling process;

- Exposure scenario to the consumer.

Regarding the input concentration, EFSA assumes a concentration of $3 \mathrm{mg} / \mathrm{kg}$ as worst-case input concentration for washed PET beverage bottle flakes. This value was derived from a Europe-wide screening of post-consumer PET flake samples [14]. Within this project, 689 samples of PET flakes from commercial washing plants were collected from 12 European countries. These samples were analysed due to their contamination levels. As a result, average levels of contaminants such as misuse chemicals such as solvents ranged from 1.4 to $2.7 \mathrm{mg} / \mathrm{kg}$, and statistically were shown to result from 0.03 to $0.04 \%$ of recollected PET bottles showing hints of misuse, which means that the these bottles might be used for storage of solvents of household and garden chemicals.

The cleaning efficiency of the mechanical recycling process should be in a position to reduce the contamination to concentration levels that do not pose a risk to human health. Starting from this average worst-case concentration of $3 \mathrm{mg} / \mathrm{kg}$, the mechanical recycling process must end up below the maximum bottle wall concentration established by the exposure scenario (see below). This must be shown within a so-called challenge test by 
artificial contamination of the input material (washed flakes) and subsequently recycling with the recycling process.

Regarding the exposure to consumers, the EFSA concluded that $0.0025 \mu \mathrm{g}$ per $\mathrm{kg}$ body weight per day for an unknown contaminant represents a negligible risk to the consumer. This value is coming from the Threshold of Toxicological Concern (TTC) Concept and includes genotoxic compounds [15]. In addition, the authority assumes that an infant with $5 \mathrm{~kg}$ body weight drinks $0.75 \mathrm{~L}$ water per day from a PET bottle made from $100 \%$ recyclate. This results in a maximum migration of $0.017 \mu \mathrm{g} / \mathrm{L}$ from a recyclate containing PET bottle into mineral water. This is a very low migration value and will be hard to determine for all of the applied model substances within the challenge test. Therefore, the EFSA uses migration modelling for the calculation of the bottle wall concentrations corresponding to the maximum migration of $0.017 \mu \mathrm{g} / \mathrm{L}$. Due to the fact that the applied migration model over-estimates the real migration by a factor of at least 5 , the maximum tolerable migration value is set to $0.1 \mu \mathrm{g} / \mathrm{L}$ [13]. This migration value should not be exceeded at the end of the shelf life of $365 \mathrm{~d}$ at room temperature $\left(25^{\circ} \mathrm{C}\right)$. These modelled maximum bottle wall concentrations can therefore be considered as the maximum values allowed in PET bottles manufactured from $100 \%$ mechanically recycled post-consumer recyclate.

In principle, similar evaluation criteria should be applied for chemical recycling processes. However, for chemical recycling processes, such evaluation criteria are not available to date. The lack of evaluation criteria is most probably due to the fact that chemical recycling processes are not within the scope of the European Recycling Regulation $282 / 2008$ [16]. This regulation covers only mechanical recycling processes that produce food-grade recyclates for their application in direct contact to food. Therefore, EFSA is at the moment not responsible for the safety evaluation of chemical recycling processes. Currently, it is assumed that cleaning efficiencies of chemical recycling processes is so high that the chemical recycling process can be considered as safe. However, in order not to distort competition, within a forthcoming amendment of the Recycling Regulation, chemical recycling processes might be also be subjected to comprehensive evaluation by EFSA, similar to that of mechanical recycling processes.

The aim of this study was to determine the cleaning efficiency of the investigated polyethylene terephthalate chemical recycling process based on the depolymerisation of PET and the repolymerisation to PET. On the basis of these cleaning efficiency data, this study evaluated the safety of chemical recycling processes. Even if chemical recycling processes are not evaluated by EFSA, their evaluation approach for mechanically recycled polymers was adapted and used for the evaluation of chemically recycled PET.

\section{Materials and Methods}

\subsection{Artificially Contaminated PET}

The cleaning efficiency is usually determined by a so-called challenge test by artificial contamination of PET flakes. Post-consumer PET flakes were contaminated with the surrogates chloroform, toluene, chlorobenzene, phenyl cyclohexane, methyl salicylate, benzophenone, and methyl stearate (Table 1). The contaminated flakes were subsequently fed into the investigated chemical recycling process. It should be noted that the artificially contaminated PET flakes were introduced into the chemical recycling process without any washing step.

\subsection{Depolimerisation of Contaminated PET Flakes}

Under inert conditions, a $1 \mathrm{~L}$ three-neck round-bottom flask was charged with $120 \mathrm{~g}$ of artificially contaminated PET, $700 \mathrm{~mL}$ ethylene glycol, and $0.687 \mathrm{~g}$ zinc acetate dehydrate as catalyst $(0.5 \mathrm{~mol} \%$ on PET). After $4 \mathrm{~h}$ of reflux while stirring with a magnetic stirrer bar (around $195-196^{\circ} \mathrm{C}$ ), the mixture was allowed to cool to a temperature of $100-150{ }^{\circ} \mathrm{C}$, upon which the unreacted material was filtered off to remove minor amounts of unreacted residues. After further cooling of the liquid under $100^{\circ} \mathrm{C}, 1 \mathrm{~L}$ of water and $1.8 \mathrm{~g}$ of active carbon were added to the mixture, which was then kept for $40 \mathrm{~min}$ at $90{ }^{\circ} \mathrm{C}$ prior to hot 
vacuum filtration over a 0.4 micron membrane filter. Subsequently, another $1 \mathrm{~L}$ of water was added to the filtrate, and it was cooled at $4{ }^{\circ} \mathrm{C}$ for $16 \mathrm{~h}$, after which it was filtered over a glass filter of pore size $40-100 \mu \mathrm{m}$ at ambient temperature. The residue was dried at $70^{\circ} \mathrm{C}$ for $24 \mathrm{~h}$ in order to yield a white, needle-like powder of bis-hydroxyethyl terephthalate (BHET). The procedure described above was repeated six times to obtain a quantity of BHET sufficient for repolymerisation.

Table 1. Model contaminants for the determination of the cleaning efficiencies (challenge test).

\begin{tabular}{cccc}
\hline Surrogate & MW & Functional Group & Physical Properties \\
\hline Toluene & 92.1 & aromatic hydrocarbon & volatile, non-polar \\
Chlorobenzene & 112.6 & halogenated aromatic hydrocarbon & volatile, medium-polar, aggressive to PET \\
Chloroform & 119.4 & halogenated hydrocarbon & volatile, polar, aggressive towards PET \\
Methyl salicylate & 152.2 & aromatic ester & non-volatile, polar \\
Phenyl cyclohexane & 160.3 & aromatic hydrocarbon & non-volatile, polar \\
Benzophenone & 182.2 & aromatic ketone & non-volatile, polar \\
Methyl stearate & 298.5 & aliphatic ester & \\
\hline
\end{tabular}

${ }^{1}$ Molecular weight in $\mathrm{g} / \mathrm{mol}$.

\subsection{Repolimerisation of BHET}

A $1 \mathrm{~L}$ cylindrical glass reactor equipped with an overhead stirrer, a vacuum outlet with cold trap, a heating jacket, an internal thermocouple, and a bottom discharge nozzle was charged with $660 \mathrm{~g}$ BHET and brought under inert conditions with nitrogen. The jacket temperature was set to $245^{\circ} \mathrm{C}$, and the BHET was given $1.5 \mathrm{~h}$ to melt and heat up to $220^{\circ} \mathrm{C}$ internal temperature, during which the overhead stirrer was started after approximately $1 \mathrm{~h}$. Subsequently, the reactor was charged with $300 \mathrm{mg} / \mathrm{kg}(w / w) \mathrm{Sb}$ catalyst as a solution of $1.25 \%(w / w) \mathrm{Sb}_{2} \mathrm{O}_{3}$ in ethylene glycol, and the jacket temperature was ramped up linearly to $293^{\circ} \mathrm{C}$ over $45 \mathrm{~min}$, during which vacuum was applied stepwise from 500 to 200, 100, 10 , and 0.5 mbar. These conditions of $293{ }^{\circ} \mathrm{C}$ jacket temperature, stirring, and $0.5 \mathrm{mbar}$ vacuum were maintained for $1.5 \mathrm{~h}$ to complete melt polycondensation under continuous removal of ethylene glycol in the cold trap, during which the product temperature levelled off and remained at about $280^{\circ} \mathrm{C}$ for the last $1 \mathrm{~h}$. Then, the product was discharged and pelletised, followed by crystallisation and solid-state polycondensation (SSP) in a vacuum oven at 0.3 to 0.5 mbar. For this step, the pellets were spread evenly on a single tray and allowed to crystallise for $4 \mathrm{~h}$ at $130{ }^{\circ} \mathrm{C}$, to dry for $2 \mathrm{~h}$ at $160{ }^{\circ} \mathrm{C}$, and to undergo SSP for $3 \mathrm{~h}$ at $190^{\circ} \mathrm{C}$, followed by $10 \mathrm{~h}$ at $234^{\circ} \mathrm{C}$ oven temperatures. The final intrinsic viscosity (IV) of the pellets was measured at $0.779 \mathrm{dL} / \mathrm{g}$ according to ASTM D4603 [17].

\subsection{Determination of the Concentrations of the Model Substance in PET and BHET}

A total of $1.0 \mathrm{~g}$ of each PET and BHET sample was analysed twice as follows. The sample was weighed into a $5 \mathrm{~mL}$ glass vial. A total of $1.0 \mathrm{~mL}$ 1,1,1,3,3,3-hexafluoro-isopropanol (HFIP) was given to the PET material and stored for $1 \mathrm{~d}$ at $60^{\circ} \mathrm{C}$ in order to swell the PET matrix or dissolve the BHET sample. Then, $2.0 \mathrm{~mL}$ iso-propanol was added, and the mixture was stored for an additional $1 \mathrm{~d}$ at $60^{\circ} \mathrm{C}$. The extract was decanted from the sample and stored for $8 \mathrm{~h}$ at $4{ }^{\circ} \mathrm{C}$. Subsequently, the extracts were decanted again from the precipitate and analysed by gas chromatography with flame ionisation detector (FID) and electron capture detector (ECD). For this purpose, a gas chromatograph with a simultaneous detection by an FID and ECD detector was used. Quantification was achieved by external calibration using the standard addition method. Parts of a standard solution of the surrogates in iso-propanol were added to uncontaminated PET flakes and were analysed together with the PET samples of the contamination experiments. Gas chromatograph: HP 5890II, column: SE $10-30 \mathrm{~m}-0.32 \mathrm{~mm}$ i.d. $-0.32 \mu \mathrm{m}$ film thickness, temperature program: $40{ }^{\circ} \mathrm{C}(5 \mathrm{~min})$, rate $15^{\circ} \mathrm{C} / \mathrm{min}, 240{ }^{\circ} \mathrm{C}(15 \mathrm{~min})$, pressure: $50 \mathrm{kPa}$ hydrogen, split: $10 \mathrm{~mL} / \mathrm{min}$. The detection limits were determined according to DIN 32645 [18]. 


\subsection{Diffusion Modelling}

Diffusion modelling was performed using the AKTS SML software version 4.54 (AKTS AG, Siders, Switzerland). The program uses finite element analysis [19]. The modelling parameters for PET are given in [20]. The following parameters were used for the calculation: $A_{P}=3.1, \tau=1577 \mathrm{~K}, \mathrm{~K}_{\mathrm{P}, \mathrm{F}}=1$. In addition, it was assumed that $1 \mathrm{~kg}$ of food was packed with an area of $6 \mathrm{dm}^{2}$ (EU cube). The storage condition of $365 \mathrm{~d}$ at $25^{\circ} \mathrm{C}$ was used. The density of PET was assumed to $1.4 \mathrm{~g} / \mathrm{cm}^{3}$. A bottle wall thickness of $200 \mu \mathrm{m}$ was used for the calculations. The modelling conditions were similar to the EFSA evaluation of mechanically recycled PET [13].

\section{Results and Discussion}

\subsection{Inut Contamination Levels}

The input contamination levels are one of the main criteria for the evaluation of recycling processes. The contamination in the feedstock of chemical processes can be significantly higher and more inhomogeneous compared to PET flakes from beverage bottles. Most chemical recycling processes target hard-to-recycle packaging such as thermoformed trays and coloured or non-food bottles, and some are developing processes using fibres (clothing, carpets) as input. For PET containers used for non-food applications, the contamination levels were recently published [21]. Within this study, individual PET containers were investigated, which were used for non-food packaging applications, e.g., for dishwashing agents, antifreeze products, mouth-washing liquids, sanitary products, shower gels, soap, and shampoos. The containers were analytically screened for substances from the first use and those that are absorbed into the PET. As a result, the concentrations of solvents were determined up to $1100 \mathrm{mg} / \mathrm{kg}$ in the washed PET, especially for solvents such as ethanol. Flavour substances were detected in concentrations of up to $100 \mathrm{mg} / \mathrm{kg}$. These concentrations can be assumed as input contamination levels in non-food containers. On the other hand, only a very limited number of samples was analysed in the study. Thus, the input contamination levels of containers from non-food applications were only roughly determined. To the knowledge of the author, other contamination profiles for the input of chemical recycling processes, especially for carpets and clothing, are not given in the scientific literature. Due to the heterogeneity of these input materials, it will be most probably hard to determine the input contamination levels, which might be the reason for the lack of data. However, from the limited amount of data available, it can be concluded that the contamination levels of the input materials for chemical recycling processes will be much higher as for washed bottles flakes from beverage applications. In addition, more substances will be most probably detectable due to the broader range of input materials compared to mechanical recycling processes. Therefore, the input contamination level of $3 \mathrm{mg} / \mathrm{kg}$, which is used for the evaluation of mechanical recycling processes [13], will be not applicable for chemical recycling processes. The concentrations are most probably much higher but can be only roughly estimated at the moment.

\subsection{Cleaning Efficiency of the Chemical Process}

The cleaning efficiency of the depolymerisation process was determined by use of a so-called challenge test. Within this challenge test, PET flakes were artificially contaminated with model substances (so-called surrogates). The contaminated material was subsequently depolymerised to bis(2-hydroxyethyl) terephthalate (BHET). Subsequently, BHET was repolymerised to amorphous PET, which was further crystallised and treated with the solid-state polycondensation process. BHET, as well as amorphous and crystallised PET pellet samples, were analysed regarding their concentration levels of the model substances in order to determine the cleaning efficiency of the chemical recycling process (Table 2). The results show that the depolymerisation process very efficiently removed all model substances. The concentrations of all applied model substances were below the analytical detection limits. For all substances, cleaning efficiencies of the depolymerisation step of $>99.9 \%$ were determined. Due to the fact that all model substances were below the 
detection limits after depolymerisation, the cleaning efficiency of the repolymerisation process cannot be derived within this study. However, the cleaning efficiency of a similar PET repolymerisation process was published in the scientific literature [22]. The applied surrogates were the same as in this study, but the high molecular weight substance lindane (hexachlorocyclohexane) was additionally added to the surrogate mixture. The neat mixture of the surrogates was pumped into a mixture of the molten PET pre-polymer and depolymerised PET flakes [22]. Such a direct contamination procedure allows very high concentration levels in the challenge test, and different contamination levels can be easily applied. Following this direct contamination procedure, the cleaning efficiency of the repolymerisation process was determined. As a result, the cleaning efficiencies of all applied substances were in any case $>99.9 \%$, with starting concentrations up to $3000 \mathrm{mg} / \mathrm{kg}$. This indicates that the investigated repolymerisation process [22] also has a very high cleaning efficiency that removes chemical contaminants efficiently by use of the applied vacuum and the high temperatures in the melt phase. Thus, as an overall result, the cleaning efficiency of both processes, the depolymerisation process, and the repolymerisation process have cleaning efficiencies of $>99.9 \%$.

Table 2. Concentrations of the applied model substances before and after depolymerisation (challenge test).

\begin{tabular}{ccccc}
\hline & \multicolumn{2}{c}{ Concentration (mg/kg) (Cleaning Efficiency) } \\
\hline & $\begin{array}{c}\text { Contaminated PET Flakes } \\
\text { before Depolymerisation }\end{array}$ & $\begin{array}{c}\text { BHET after } \\
\text { Depolymerisation }\end{array}$ & $\begin{array}{c}\text { Amorphous PET } \\
\text { Pellets after } \\
\text { Repolymerisation }\end{array}$ & $\begin{array}{c}\text { Crystallised PET Pellets } \\
\text { after Solid-Stating }\end{array}$ \\
\hline Toluene & $157.7 \pm 8.7$ & $<0.10(>99.94 \%)$ & $<0.10$ & $<0.10$ \\
Chlorobenzene & $733.9 \pm 313.0$ & $<0.03(>99.99 \%)$ & $<0.03$ & $<0.03$ \\
Chloroform & $485.1 \pm 233.2$ & $<0.10(>99.98 \%)$ & $<0.10$ & $<0.10$ \\
Methyl salicylate & $852.6 \pm 297.2$ & $<0.04(>99.99 \%)$ & $<0.04$ & $<0.04$ \\
Phenyl cyclohexane & $720.6 \pm 507.2$ & $<0.05(>99.99 \%)$ & $<0.05$ & $<0.05$ \\
Benzophenone & $712.5 \pm 352.6$ & $<0.06(>99.99 \%)$ & $<0.06$ & $<0.04$ \\
Methyl stearate & $865.6 \pm 507.5$ & $<0.04(>99.99 \%)$ & $<0.04$ & $<$ \\
\hline
\end{tabular}

\subsection{Safety Evaluation of a PET Chemical Recycling Process}

As mentioned above, the cleaning efficiencies of the depolymerisation process as well as for the repolymerisation process were both above $99.9 \%$ for all applied surrogates, which resulted in a very high cleaning efficiency of $99.9999 \%$ for the overall chemical recycling process. Due to this extremely high cleaning efficiency, it is nearly impossible to determine experimentally the cleaning efficiency of the overall process because the necessary artificial input contamination needs to be either too high or the detection limits in the final product of the chemical recycling process needs to be very low. As a consequence, the cleaning efficiencies of both the depolymerisation process and the repolymerisation process needs to be determined individually in two separate challenge tests.

On the other hand, the input contamination levels are also only roughly known, but most probably much higher than $3 \mathrm{mg} / \mathrm{kg}$ used for the evaluation of PET recyclates from mechanical recycling processes [13]. Due to the lack of information on the input contamination levels, a fixed starting level for the contamination in the input materials for chemical recycling processes is not suitable for the evaluation of the safety of chemical recycling processes. However, without a fixed input contamination, the evaluation criteria published by the EFSA for mechanical recycling processes are not applicable for chemical recycling processes.

A reversed approach seems to be more suitable for the safety evaluation of chemical recycling processes. Within this approach, cleaning efficiencies are not applied to a fixed input contamination level. Instead, the concentration in a PET mineral water bottle was calculated, which corresponded to a migration level of $0.1 \mu \mathrm{g} / \mathrm{L}$ after storage for $365 \mathrm{~d}$ at $25^{\circ} \mathrm{C}$. This migration level as well as the storage conditions had been used for the evaluation of mechanically recycled PET by EFSA [13]. These maximum concentrations 
in the PET bottle were calculated for the substances typically used in challenge test, but also for other substances with defined molecular weights up to $1000 \mathrm{~g} / \mathrm{mol}$. The results of the maximum bottle wall concentrations corresponding with a migration level of $0.1 \mu \mathrm{g} / \mathrm{L}$ after storage for $365 \mathrm{~d}$ at $25^{\circ} \mathrm{C}$ are given in Table 3. On the first approach, these maximum concentrations in the bottle wall were calculated without consideration of any cleaning efficiencies of the depolymerisation and repolymerisation process. This means that the pure migration process had been considered in the concentration given in Table 3. Assuming cleaning efficiencies of $90 \%, 99 \%$, and $99.9 \%$ the maximum concentrations for each increased by a factor of 10,100, and 1000, respectively (Table 3, Figure 1).

Table 3. Calculated maximum concentrations corresponding to a migration of $0.1 \mu \mathrm{g} / \mathrm{L}$ after storage for $365 \mathrm{~d}$ at $25^{\circ} \mathrm{C}$ (EU cube, $\mathrm{A}_{\mathrm{P}}=3.1, \tau=1577 \mathrm{~K}$, bottle wall thickness $200 \mu \mathrm{m}$, density of PET $\left.1.4 \mathrm{~g} / \mathrm{cm}^{3}\right)$.

\begin{tabular}{|c|c|c|c|c|c|}
\hline \multirow[t]{2}{*}{ Surrogate } & \multirow[t]{2}{*}{ MW $^{1}$} & \multicolumn{4}{|c|}{$\begin{array}{l}\text { Maximum Bottle Wall Concentration }(\mathrm{mg} / \mathrm{kg}) \\
\text { Considering the Cleaning Efficiency }\end{array}$} \\
\hline & & $0 \%$ & $90 \%$ & $99 \%$ & $99.9 \%$ \\
\hline Toluene & 92.1 & 0.0797 & 0.797 & 7.97 & 79.7 \\
\hline Chlorobenzene & 112.6 & 0.0945 & 0.945 & 9.45 & 94.5 \\
\hline Chloroform & 119.4 & 0.0990 & 0.990 & 9.90 & 99.0 \\
\hline Methyl salicylate & 152.2 & 0.126 & 1.26 & 12.6 & 126 \\
\hline Phenyl cyclohexane & 160.3 & 0.133 & 1.33 & 13.3 & 133 \\
\hline Benzophenone & 182.2 & 0.154 & 1.54 & 15.4 & 154 \\
\hline Methyl stearate & 298.5 & 0.300 & 3.00 & 30.0 & 300 \\
\hline Fictive & 400 & 0.495 & 4.95 & 49.5 & 495 \\
\hline Fictive & 500 & 0.770 & 7.70 & 77.0 & 770 \\
\hline Fictive & 750 & 1.98 & 19.8 & 198 & 1980 \\
\hline Fictive & 1000 & 4.41 & 44.1 & 441 & 4410 \\
\hline
\end{tabular}

${ }^{1}$ Molecular weight in $\mathrm{g} / \mathrm{mol}$.

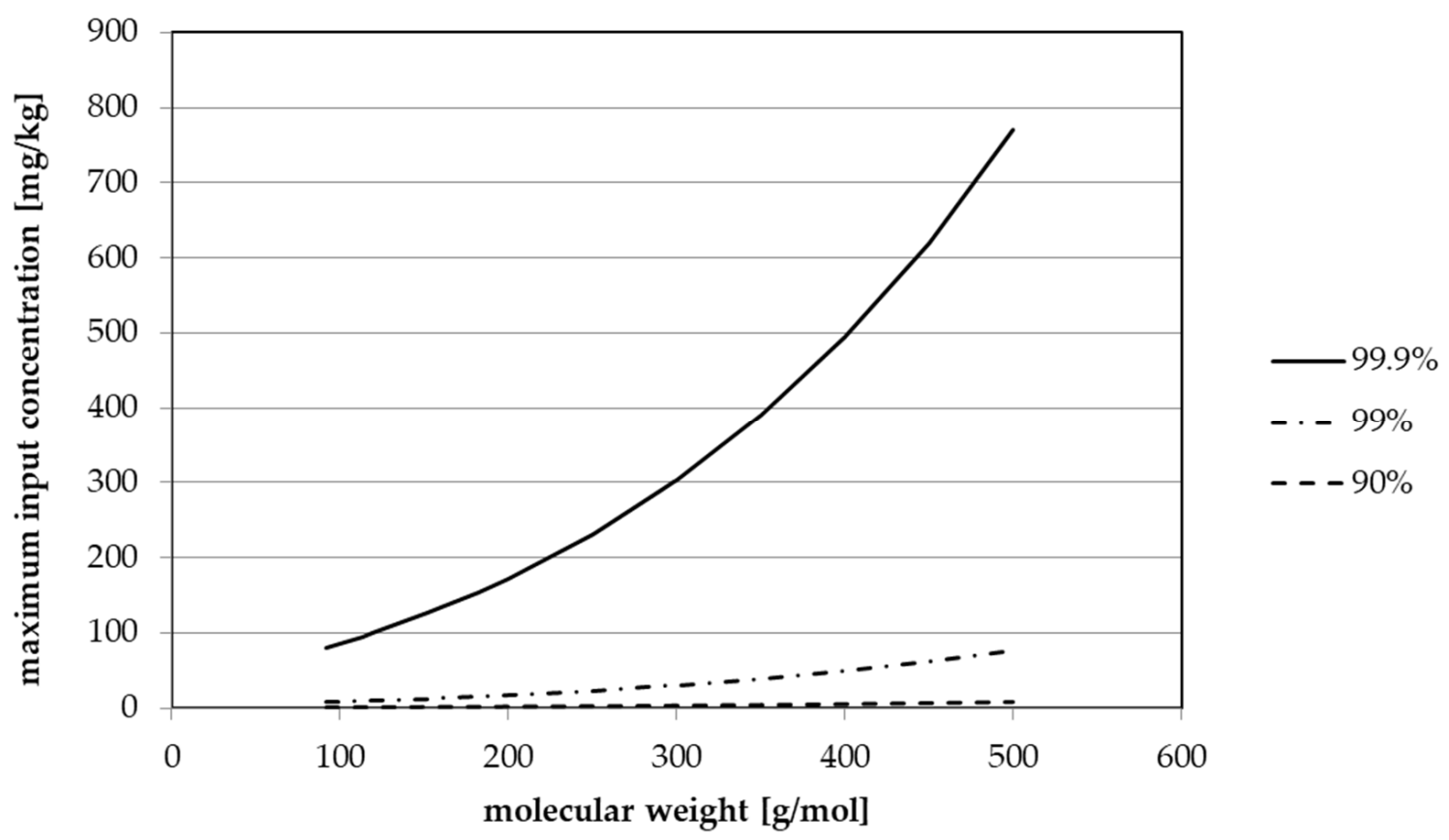

Figure 1. Maximum input contamination levels in the feedstock of chemically recycled PET for assumed cleaning efficiencies of $99.9 \%, 99 \%$, and $90 \%$. Data from Table 3 .

As an example, toluene as the lowest molecular weight substance in the surrogate mixture showed the highest migration. A concentration of $0.0797 \mathrm{mg} / \mathrm{kg}$ in PET resulted in a migration of $0.1 \mu \mathrm{g} / \mathrm{L}$ after a storage time of $365 \mathrm{~d}$ at $25^{\circ} \mathrm{C}$. If a cleaning efficiency of 
$0 \%$ is assumed, this concentration is the maximum tolerable concentration of toluene (or a substance with similar molecular weight) in the input material for the depolymerisation process. Assuming a cleaning efficiency of $99 \%$, this maximum concentration in the input material increases by a factor of 100 to $7.97 \mathrm{mg} / \mathrm{kg}$. With a cleaning efficiency of $99.9 \%$, the maximum concentration in the input material is $79.7 \mathrm{mg} / \mathrm{kg}$. Assuming that the repolymerisation process also has a cleaning efficiency of $99.9 \%$, the maximum concentration of toluene increases by an additional factor of 1000 to $79,700 \mathrm{mg} / \mathrm{kg}$, which is a concentration of $7.97 \%$ of toluene in the input material of a chemical recycling process.

With higher molecular weights of the applied substances, the maximum input concentrations increases up to $770 \mathrm{mg} / \mathrm{kg}$ (with $99.9 \%$ cleaning efficiency) for a (fictive) substance with a molecular weight if $500 \mathrm{~g} / \mathrm{mol}$ (Table 3). Again, the (maximum) input contamination level of input streams for chemical recycling process will be hard to determine. However, when only the repolymerisation process is considered, which started from the monomers or oligomers after depolymerisation, the situation will be much easier. In terms of the monomers or oligomers, the purification level is much easier to determine and is moreover part of the production control of a chemical recycling process. These maximum concentration levels in monomers and oligomers can be analytically controlled as a routine test. This will be much easier, and the evaluation of a chemical recycling can be independent from the depolymerisation process.

The high cleaning efficiencies are in agreement with the preamble of the Recycling Regulation 282/2008 [16], where it is mentioned that "monomers and oligomers resulting from chemical depolymerisation should not be treated differently from monomers manufactured by chemical synthesis. Therefore, they are covered by the authorisation of monomers and additives in Directive 2002/72/EC [remark, now Regulation 10/2011] and they should comply with the specifications and purity criteria established therein". This means that the same purity requirements apply as for virgin polymers without recyclate content. In addition, the U.S. Food and Drug Administration eliminated all recommendations for chemical recycling processes in the actual guidance document, and the FDA no longer recommends that such recyclers submit data for agency evaluation [23]. The FDA concludes that "recycling of PET or PEN by methanolysis or glycolysis results in the production of monomers or oligomers that are readily purified to produce a finished polymer that is suitable for food-contact use".

\section{Conclusions}

The results of this study show that the overall cleaning efficiency of a chemical PET recycling process are so high that the cleaning efficiency can hardly be tested within one challenge test. The process steps, depolymerisation and repolymerisation, need to be tested in two separate challenge tests in order to determine the cleaning efficiencies of each of these two parts. When only the second part of the recycling process, the repolymerisation of PET, is considered, the cleaning efficiencies were $>99.9 \%$ for all applied model substances. Such a high cleaning efficiency is enough to show that the overall chemical recycling process can be considered as safe. The second important piece of information for the evaluation of the process, the initial concentration in the feedstock material of a chemical PET recycling process, can only be roughly estimated due to the heterogeneous input material for chemical recycling processes. Therefore, the evaluation principle for PET mechanical recycling processes published by EFSA is not applicable for chemical recycling processes. Uncertainties in the determination of the input concentrations might be the major drawback for the circular economy. This seems to also be a problem for mechanical recycling processes where the recollection and input streams are not established in industrial scale. Without an industrial scale input stream, a statistically validated input concentration can be hardly determined, and the mechanical recycling process cannot be evaluated according to the EFSA criteria, even if the cleaning efficiency of the recycling process is available. This seems to be a chicken and egg problem. 
The reversed approach applied in this study might be a solution to this dilemma. If the input contamination levels are not available, this reversed approach calculates the maximum input concentrations, which are in compliance with the existing threshold limits set by the EFSA. Within the study, the maximum concentrations in the depolymerised monomers and oligomers were calculated in a generic approach by using different molecular weight substances up to $1000 \mathrm{~g} / \mathrm{mol}$ as well as by the use of different cleaning efficiencies. These concentrations can be considered as the maximum input contamination levels in the starting materials for the repolymerisation process. By using this generic approach, these maximum input contamination levels are at first glance independent of the repolymerisation technology. However, when knowing the cleaning efficiencies of an individual repolymerisation process, the maximum input concentrations can be derived from this generic approach. As long as the input contamination levels are below these maximum tolerable concentrations, the process can be considered as safe. If the repolymerisation process alone is able to guaranty the safety of the chemical recycling process, the cleaning efficiency of the depolymerisation process need not to be determined and provides only additional safety factors when the cleaning efficiency is known. When this approach is followed, the initial concentrations of the depolymerisation process need no longer to be considered in the safety evaluation of chemical recycling processes, which simplifies the assessment of the overall chemical recycling process considerably. Only the cleaning efficiencies of the repolymerisation process have to be determined by use of a challenge test.

A major advantage of this reverse approach is that these maximum contamination levels can be easily controlled in the routine quality control tests of a chemical recycling plant. These maximum input contaminant levels are clear target concentrations for the quality assurance of the monomers derived from chemical recycling processes.

Funding: This research received no external funding.

Institutional Review Board Statement: Not applicable.

Informed Consent Statement: Not applicable.

Data Availability Statement: The data used to support the findings of this study are included within the article.

Acknowledgments: The author would like to thank Anita Gruner and Alexandra Mauer, both Fraunhofer IVV, for experimental support of this study.

Conflicts of Interest: The author declares no conflict of interest.

\section{References}

1. Lugal, L.; Grant, A.; Cordle, M.; Fletcher, D. PET Market in Europe: State of Play. Eunomia Reports 2020. Available online: https:/ / www.eunomia.co.uk/reports-tools/pet-market-in-europe-state-of-play / (accessed on 14 October 2021).

2. Platt, P. The Future of PET Packaging to 2025; Smithers Information Ltd.: Akron, OH, USA, 2020.

3. Shojaei, B.; Abtahi, M.; Najafi, M. Chemical recycling of PET: A stepping-stone toward sustainability. Polym. Adv. Technol. 2020, 31, 2912-2938. [CrossRef]

4. Welle, F. Twenty Years of PET Bottle to Bottle Recycling-An Overview. Res. Conserv. Recycl. 2011, 55, 865-875. [CrossRef]

5. Van Velzen, E.U.T.; Brouwer, M.T.; Stärker, C.; Welle, F. The effect of recycled content and rPET quality on the properties of PET bottles, Part II: Migration. Pack. Techn. Sci. 2020, 33, 359-371. [CrossRef]

6. Lechleitner, A.; Schwabl, D.; Schubert, T.; Bauer, M.; Lehner, M. Chemical recycling of mixed waste plastics as complementary process pathway to increase recycling rates. Österr Wasser-Abfallw. 2020, 72, 47-60. [CrossRef]

7. Chaudhari, U.S.; Lin, Y.; Thompson, V.S.; Handler, R.M.; Pearce, J.M.; Caneba, G.; Muhuri, P.; Watkins, D.; Shonnard, D.R. Systems analysis approach to polyethylene terephthalate and olefin plastics supply chains in the circular economy: A review of data sets and models. ACS Sustain. Chem. Eng. 2021, 9, 7403-7421. [CrossRef]

8. Park, S.H.; Kim, S.H. Poly (ethylene terephthalate) recycling for high value added textiles. Fash. Text. 2014, 1, 1. [CrossRef]

9. Sinha, V.; Patel, M.R.; Patel, J.V. PET waste management by chemical recycling: A review. J. Polym. Environ. 2010, 18, 8-25. [CrossRef]

10. Geyer, B.; Röhner, S.; Lorenz, G.; Kandelbauer, A. Designing oligomeric ethylene terephthalate building blocks by chemical recycling of polyethylene terephtalate. J. Appl. Polym. Sci. 2014, 131, 1-12. [CrossRef] 
11. Payne, J.; Jones, M.D. The chemical recycling of polyesters for a circular plastics economy: Challenges and emerging opportunities. ChemSusChem 2021, 14, 4041-4070. [CrossRef] [PubMed]

12. Welle, F. Polyethylene terephthalate (PET)—Prime material for a circular economy. Kunstst. Int. 2019, 109, 51-54.

13. EFSA Panel on Food Contact Materials, Enzymes, Flavourings and Processing Aids (CEF). Scientific Opinion on the criteria to be used for safety evaluation of a mechanical recycling process to produce recycled PET intended to be used for manufacture of materials and articles in contact with food. EFSA J. 2011, 9, 2184.

14. Franz, R.; Mauer, A.; Welle, F. European survey on post-consumer poly(ethylene terephthalate) materials to determine contamination levels and maximum consumer exposure from food packages made from recycled PET. Food Add. Contam. 2004, 21, 265-286. [CrossRef] [PubMed]

15. Kroes, R.; Renwick, A.G.; Feron, V.; Galli, C.L.; Gibney, M.; Greim, H.; Guy, R.H.; Lhuguenot, J.C.; van de Sandt, J.J.M. Application of the threshold of toxicological concern (TTC) to the safety evaluation of cosmetic ingredients. Food Chem. Toxicol. 2007, 45, 2533-2562. [CrossRef] [PubMed]

16. Official Journal of the European Union. Commission Regulation (EC) No 282/2008 of 27 March 2008 on recycled plastic materials and articles intended to come into contact with foods and amending Regulation (EC) No 2023/2006. Off. J. Eur. Union 2008, L86/9. Available online: https:/ / eur-lex.europa.eu/LexUriServ/LexUriServ.do?uri=OJ:L:2008:086:0009:0018:EN:PDF (accessed on 16 November 2021).

17. ASTM D4603-18. Standard Test Method for Determining Inherent Viscosity of Poly(Ethylene Terephthalate) (PET) by Glass Capillary Viscometer. Developed by Subcommittee: D20.70. Available online: https://www.astm.org/Standards/D4603.htm (accessed on 16 November 2021).

18. DIN 32645:2008-11. Chemical Analysis—Decision Limit, Detection Limit and Determination Limit under Repeatability Conditions-Terms, Methods, Evaluation; Beuth Verlag: Berlin, Germany, 2011. Available online: https://www.beuth.de/de/norm/din-32645/110729 574 (accessed on 16 November 2021).

19. Roduit, B.; Borgeat, C.H.; Cavin, S.; Fragniere, C.; Dudler, V. Application of finite element analysis (FEA) for the simulation of release of additives from multilayer polymeric packaging structures. Food Addit. Contam. Part A 2005, 22, 945-955. [CrossRef] [PubMed]

20. Brandsch, R.; Dequatre, C.; Mercea, P.; Milana, M.-R.; Störmer, A.; Trier, X.; Vitrac, O.; Schäfer, A.; Simoneau, C. Practical Guidelines on the Application of Migration Modelling for the Estimation of Specific Migration; EU Report 27529 EN; 2015; ISBN 978-92-79-52790-6. Available online: https:/ / op.europa.eu/de/publication-detail/-/publication/1b79bc61-97f6-11e5-983e-01aa75ed71a1 (accessed on 16 November 2021).

21. Franz, R.; Welle, F. Contamination levels in re-collected PET bottles from non-food applications and their impact on the safety of recycled PET for food contact. Molecules 2020, 25, 4998. [CrossRef] [PubMed]

22. Welle, F. Decontamination efficiency of a new post-consumer poly(ethylene terephthalate) (PET) recycling concept. Food Addit. Contam. 2008, 25, 123-131. [CrossRef] [PubMed]

23. FDA. Use of Recycled Plastics in Food Packaging (Chemistry Considerations): Guidance for Industry. 2021. Available online: https: / / www.fda.gov / media/150792/download (accessed on 24 October 2021). 